\title{
Distribution Locational Marginal Pricing through Quadratic Programming for Congestion Management in Distribution Networks
}

\author{
Huang, Shaojun; Wu, Qiuwei; Oren, Shmuel S.; Li, Ruoyang; Liu, Zhaoxi
}

Published in:

IEEE Transactions on Power Systems

Link to article, DOI:

10.1109/TPWRS.2014.2359977

Publication date:

2015

Document Version

Peer reviewed version

Link back to DTU Orbit

Citation (APA):

Huang, S., Wu, Q., Oren, S. S., Li, R., \& Liu, Z. (2015). Distribution Locational Marginal Pricing through Quadratic Programming for Congestion Management in Distribution Networks. IEEE Transactions on Power Systems, 30(4), 2170 - 2178. https://doi.org/10.1109/TPWRS.2014.2359977

\section{General rights}

Copyright and moral rights for the publications made accessible in the public portal are retained by the authors and/or other copyright owners and it is a condition of accessing publications that users recognise and abide by the legal requirements associated with these rights.

- Users may download and print one copy of any publication from the public portal for the purpose of private study or research.

- You may not further distribute the material or use it for any profit-making activity or commercial gain

- You may freely distribute the URL identifying the publication in the public portal 


\title{
Distribution Locational Marginal Pricing through Quadratic Programming for Congestion Management in Distribution Networks
}

\author{
Shaojun Huang, Qiuwei Wu, Member, IEEE, Shmuel S. Oren, Fellow, IEEE, Ruoyang Li, Student \\ Member, IEEE, Zhaoxi Liu
}

\begin{abstract}
This paper presents the distribution locational marginal pricing (DLMP) method through quadratic programming (QP) designed to alleviate the congestion that might occur in a distribution network with high penetration of flexible demands. In the DLMP method, the distribution system operator (DSO) calculates dynamic tariffs and publishes them to the aggregators, who make the optimal energy plans for the flexible demands. The DLMP through QP instead of linear programing as studied in previous literatures solves the multiple solution issue of the aggregator optimization which may cause the decentralized congestion management by DLMP to fail. It is proven in this paper, using convex optimization theory, the aggregator's optimization problem through QP is strictly convex and has a unique solution. The Karush-Kuhn-Tucker (KKT) conditions and the unique solution of the aggregator optimization ensure that the centralized DSO optimization and the decentralized aggregator optimization converge. Case studies using a distribution network with high penetration of electric vehicles (EVs) and heat pumps (HPs) validate the equivalence of the two optimization setups, and the efficacy of the proposed DLMP through QP for congestion management.
\end{abstract}

Index Terms-- Congestion management, distribution locational marginal pricing (DLMP), distribution system operator (DSO), electric vehicle (EV), heat pump (HP).

\section{NOMENCLATURE}

$B_{i, t} \in R^{m_{i} \times m_{i}}$ matrix of the price sensitivity coefficient

$C_{a} \quad$ heat capacity of the inside air

$C_{s} \quad$ heat capacity of the house structure (walls, etc.)

$D \in R^{n_{L} \times n_{d}} \quad$ power transfer distribution factor (PTDF)

$E_{i} \in R^{n_{d} \times m_{i}}$ customer to load bus mapping matrix

$K \quad$ outside temperature

$K^{a} \quad$ house inside temperature

$K^{s} \quad$ structure temperature

$K_{i, t}^{a, \min } \in R^{m_{i}}$ lower temperature limit

$K_{i, t}^{a, \max } \in R^{m_{i}}$ upper temperature limit

The work is supported by the EU Seventh Framework Programme (FP7) through the 'Ideal Grid for All (IDE4L)' project.

S. Huang, Q. Wu and Z. Liu are with the Center for Electric Power and Energy (CEE), Department of Electrical Engineering, Technical University of Denmark (DTU), 2800 Kgs. Lyngby, Denmark (e-mail: shuang@elektro.dtu.dk; qw@elektro.dtu.dk; zhliu@elektro.dtu.dk).

S. S. Oren and R. Li are with the Department of Industrial Engineering and Operations Research (IEOR), University of California, Berkeley, CA 94704 USA (e-mail: oren@ieor.berkeley.edu; ruoyang@berkeley.edu).

$N_{B}$
$N_{T}$
$N_{L}$
$N_{d}$
$Q^{e}$
$S_{t}^{1}$
$S_{t}^{2}$
$C_{t}$
$d_{i, t} \in R^{m_{i}}$
$e_{i}^{\min } \in R^{m_{i}}$
$e_{i}^{\max } \in R^{m_{i}}$
$e_{i, 0} \in R^{m_{i}}$
$f_{t} \in R^{n_{L}}$
$k_{1}$

set of aggregators

set of planning periods

set of lines

set of demand bus

thermal energy produced by HP

solar irradiation to the inside air

solar irradiation to the structure

baseline price

discharging power of EVs due to driving

lower limit of the state of charge (SOC) level

upper limit of the SOC level

initial SOC level

line loading limit available for flexible demands heat transfer coefficient (HTC) between the inside and the outside of the household

$k_{2} \quad$ HTC between the inside and the house structure

$k_{3} \quad$ HTC between the house structure and the outside $m_{i} \quad$ the number of customers of aggregator $i$

$n_{*} \quad$ cardinality of $N_{*}$, i.e. $n_{*}=\left|N_{*}\right|$

$p_{t} \quad$ charging power of an EV

$p_{i, t} \in R^{m_{i}} \quad$ charging power of EVs of one aggregator

$p_{i, t}^{\min } \in R^{m_{i}} \quad$ lower charging power limit of EVs

$p_{i, t}^{\max } \in R^{m_{i}} \quad$ upper charging power limit of EVs

$\hat{p}_{t} \quad$ power consumption of an HP

$\hat{p}_{i, t} \in R^{m_{i}} \quad$ power consumption of HPs of one aggregator

$\hat{p}_{i}^{\text {min }} \in R^{m_{i}} \quad$ lower power limit of HPs

$\hat{p}_{i}^{\max } \in R^{m_{i}}$ upper power limit of HPs

$y_{t} \quad$ predicted price

$\beta_{t} \quad$ price sensitivity coefficient

$\lambda_{t} \in R^{n_{L}} \quad$ Lagrange multiplier (LM) of line loading limit constraint

$\mu_{i, t}^{+} \in R^{m_{i}} \quad$ LM of SOC upper limit constraint

$\mu_{i, t}^{-} \in R^{m_{i}} \quad$ LM of SOC lower limit constraint 
$\hat{\mu}_{i, t}^{+} \in R^{m_{i}} \quad$ LM of upper temperature limit constraint

$\hat{\mu}_{i, t}^{-} \in R^{m_{i}} \quad$ LM of lower temperature limit constraint

$\varsigma_{i, t}^{+} \in R^{m_{i}} \quad$ LM of EV charging power upper limit constraint

$\varsigma_{i, t}^{-} \in R^{m_{i}} \quad$ LM of EV charging power lower limit constraint

$\hat{\zeta}_{i, t}^{+} \in R^{m_{i}} \quad$ LM of HP power upper limit constraint

$\hat{\zeta}_{i, t}^{-} \in R^{m_{i}} \quad$ LM of HP power lower limit constraint

\section{INTRODUCTION}

$\mathrm{D}$ ENMARK, as one of the countries that strive for $\mathrm{CO}_{2}$ emission reduction and energy supply security, has adopted energy strategies that aim at achieving independence from fossil fuels by 2050 [1]. In order to realize such an ambitious energy plan, renewable energy sources (RES) such as wind power (WP), solar power (SP), and distributed energy resources (DER), such as electric vehicles (EVs) and heat pumps (HPs), will be extensively used and will play an important role in the future power systems. Impacts of these new components on the power systems have been widely studied [2]-[4]. In particular, congestion problems that might occur in distribution networks due to the high penetration of DER have already drawn attention from distribution system operators (DSOs), manufacturers and researchers. A DSO, who has the main responsibility for resolving the congestion in distribution networks, can choose to reinforce the network through long term planning or employ market methods [5]-[7] so as to incentivize the DERs to respect the system capacity limits. Compared to direct control methods for congestion management [8], [9], market-based methods can maximize social welfare, cause least discomfort to customers and encourage more participation in the energy planning.

By extending the locational marginal price (LMP) concept [10] from transmission networks to distribution networks, [11]-[15] have developed the distribution LMP (DLMP) concept and applied it to handle the congestion issues in distribution networks with distributed generators (DGs). Through the DLMP concept, the local DGs will be properly subsidized if they produce more power and reduce the energy requirement at the local bus from remote areas during congestion periods.

Reference [7] employs a dynamic tariff (DT) concept, which is derived from the DLMP, to solve the congestion due to flexible demands in distribution networks. The flexible demands may create congestion if the price is not properly set; on the other hand, they can help congestion management if they are controlled through proper price signals. In [7], congestion management is implemented in a decentralized manner so that the aggregators independently determine the energy plans for flexible demands without considering network constraints. The network constraint information is contained in the DT. However, the method proposed in [7] did not consider the inter-temporal characteristics of flexible demands.

In [16], taking into account the inter-temporal characteristics, an integrated DLMP method for determining DT was proposed. The method proposed in [16] works in most cases. However, the aggregator optimization may have multiple solu- tions due to the linear programming (LP) formulation. The multiple solution issue of the aggregator optimization in the DLMP concept was discussed in [17]. Such multiple solutions may cause the centralized DSO optimization and the decentralized aggregator optimization to diverge, and the decentralized congestion management to fail.

In order to address the multiple solution issue of the decentralized aggregator optimization, this paper introduces a new quadratic programming (QP) based formulation. The contributions of this paper are: (a) Prove the existence of a unique solution to the optimization problem at both the centralized DSO side and the decentralized aggregator side, and the equivalence of these two optimizations through convex QP; (b) Demonstrate that the DLMP concept is valid with the cost function having quadratic terms reflecting price sensitivity of the DERs; (c) Demonstrate that the DLMP concept can solve congestion caused by diverse flexible demand characteristics such as, EVs and HPs.

The paper is organized as follows. Spot price prediction based on price sensitivity and optimal energy planning of EVs and HPs are presented in Section III. The non-convergence issue of the LP formulation and its resolution through QP formulation is described and analyzed in Section IV. In Section $\mathrm{V}$, case studies are presented and discussed, followed by conclusions.

\section{OPtimal ENERgy PlanNing FOR EV AND HP}

EVs and HPs meet their energy needs for driving and heating by procuring energy in the day-ahead electricity market. Such purchases can be done through an aggregator representing the EV and HP users by submitting bids on their behalf in the day-ahead electricity market. As such, the individual users shift the burden of market participation to aggregators, and the aggregators get enough capacity to participate in different markets. The day-ahead spot price prediction, and the optimal EV charging and HP planning based on the spot price prediction are explained in this section.

\section{A. Spot Price Prediction}

Before submitting their bids, the aggregators need to determine an optimal energy plan based on the predicted spot prices. The electricity prices are plan-dependent, which poses some difficulty in determining an optimal energy plan because the price is a discontinuous function of the energy plan. A price sensitivity based spot price prediction method was proposed in [18], [19] to deal with such difficulty. Specifically, the predicted price consists of a baseline price plus a linear component proportional to the demand so that the predicted spot price at time $t$ (hour) is given by,

$$
y_{t}=c_{t}+\beta_{t} p_{t}
$$

The price sensitivity coefficient $\beta$ is determined by evaluating the merit order of the power plants in the electricity market [18]. The production of renewable energy resources, such as WP and SP, is deducted from the conventional demand first. Then the net demands and the flexible demands are met by conventional power plants according to the order of their marginal cost. The function of marginal cost versus demand 
can be fit by an exponential function and $\beta$ is the first order coefficient of the Taylor expansion of the fit function. The concept of the price sensitivity is illustrated in Fig. 1. The coefficient $\beta$ estimated in the above method is scaled up by the total number of available flexible demand (EVs and HPs) in order to be used for individual flexible demand.

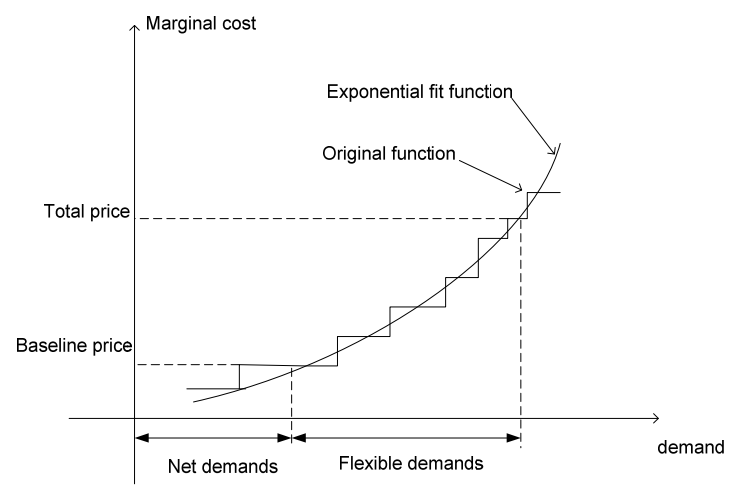

Fig. 1. Concept of the price sensitivity

\section{B. Optimal EV Charging}

The optimal EV charging aims to meet the energy needs of EVs with minimum energy cost. Taking into account the price sensitivity, the cost function of the EV charging becomes a quadratic function. The total charging cost of an EV is,

$$
\sum_{t \in N_{T}} y_{t} p_{t}=\sum_{t \in N_{T}}\left(c_{t}+\beta p_{t}\right) p_{t}=\sum_{t \in N_{T}} \beta_{t} p_{t}^{2}+c_{t} p_{t}
$$

In our framework we assume that the charging plan of the EVs managed by aggregator $i$ at period $t$ can be expressed as $p_{i, t} \in R^{m_{i}}$.

As such, the optimal EV charging plan can be found by solving the optimization problem below.

$$
\min _{p_{i, t}} \sum_{i \in N_{B}, t \in N_{T}}\left(\frac{1}{2} p_{i, t}{ }^{T} B_{i, t} p_{i, t}+\left(c_{t} 1\right)^{T} p_{i, t}\right)
$$

subject to,

$$
\begin{gathered}
e_{i}^{\min } \leq \sum_{t_{-} \leq t}\left(p_{i, t_{-}}-d_{i, t_{-}}\right)+e_{i, 0} \leq e_{i}^{\max }, \forall \mathrm{t} \in \mathrm{N}_{t}, i \in N_{B},\left(\mu_{i, t}^{-}, \mu_{i, t}^{+}\right) \\
p_{i, t}^{\min } \leq p_{i, t} \leq p_{i, t}^{\max }, \forall i \in N_{B}, t \in N_{T},\left(\varsigma_{i, t}^{-}, \varsigma_{i, t}^{+}\right)
\end{gathered}
$$

Constraint (4) ensures that the SOC levels of the batteries are within the specified range. Equations (3)-(5) form a QP problem.

\section{Optimal HP Planning}

The optimal HP planning is to schedule the energy consumption of HPs so as to maintain the house temperature within a specified range at the minimum energy cost. The heat transfer process of the air source HP can be represented by an electric circuit [20] which is illustrated in Fig. 2. Thus, the following thermal balance equations can be derived [20].

$$
\begin{gathered}
Q_{t}^{e}+S_{t}^{1}-k_{1}\left(K_{t}^{a}-K_{t}\right)-k_{2}\left(K_{t}^{a}-K_{t}^{s}\right)= \\
C_{a}\left(K_{t}^{a}-K_{t-1}^{a}\right) \forall \mathrm{t} \in N_{T}
\end{gathered}
$$

$$
\begin{gathered}
S_{t}^{2}+k_{2}\left(K_{t}^{a}-K_{t}^{s}\right)-k_{3}\left(K_{t}^{s}-K_{t}\right)= \\
C_{s}\left(K_{t}^{s}-K_{t-1}^{s}\right) \forall \mathrm{t} \in N_{T}
\end{gathered}
$$

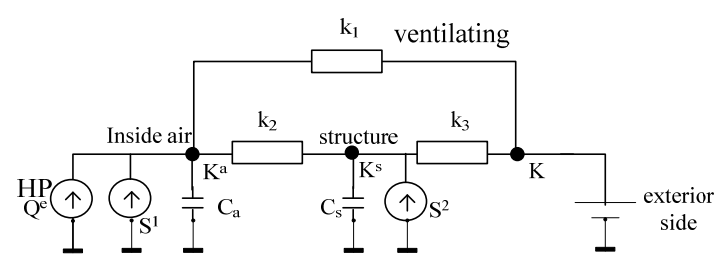

Fig. 2. Heat transferring process of the house

Equations (6) and (7) can be solved iteratively. As a result, the house inside air temperature $K_{t}^{a}$ will be a linear combination of all the previous and the current thermal energy $\left(Q_{t}^{e}\right)$ plus an initial state. Because $Q_{t}^{e}$ has a linear relation (by the coefficient of performance (COP)) to the active power $\hat{p}_{t}$ consumed by the HP, the house inside air temperature can be expressed as,

$$
K_{t}^{a}=\sum_{t_{-} \leq t} a_{t, t_{-}} \hat{p}_{t_{-}}+u_{t} \quad \forall \mathrm{t} \in N_{T}
$$

Finally, the optimization problem of the HP energy plan can be formulated as,

$$
\min _{\hat{p}_{i, t}} \sum_{i \in N_{B}, t \in N_{T}} \frac{1}{2} \hat{p}_{i, t}^{T} B_{i, t} \hat{p}_{i, t}+\left(c_{t} 1\right)^{T} \hat{p}_{i, t}
$$

subject to,

$$
\begin{gathered}
K_{i, t}^{a, \min } \leq \sum_{t_{-} \leq t} A_{i, t, t_{-}} \hat{p}_{i, t_{-}}+u_{i, t} \leq K_{i, t}^{a, \max }, \forall i \in \mathrm{N}_{B}, \mathrm{t} \in N_{T}, \\
\left(\hat{\mu}_{i, t}^{-}, \hat{\mu}_{i, t}^{+}\right) \\
\hat{p}_{i, t}^{\min } \leq \hat{p}_{i, t} \leq \hat{p}_{i, t}^{\max }, \forall i \in N_{B}, t \in N_{T},\left(\hat{\zeta}_{i, t}^{-}, \hat{\varsigma}_{i, t}^{+}\right)
\end{gathered}
$$

where $A_{i, t, t_{-}} \in R^{m_{i} \times m_{i}}$ is a diagonal matrix, $u_{i, t} \in R^{m_{i}}$.

\section{DLMP AND DT THROUGH QP}

\section{A. Decentralized Congestion Management through DLMP and $D T$}

According to [7], [16], the procedure of using the DLMP and DT concepts to solve the congestion problem in a decentralized manner can be summarized as follows. Firstly, the DSO obtains the flexible demand data, such as energy requirements and supply availability, from the aggregators or by its own prediction. The DSO also needs the distribution network information and the predicted spot prices at the relevant transmission busses. Secondly, the DLMPs are calculated through the optimal plan respecting the network constraints, and the DTs (DLMPs minus the predicted spot prices) are published to all the aggregators. Thirdly, after receiving the DTs, the aggregators make their own optimal plans independently with both the predicted spot prices and the DTs. At last, the aggregators submit their energy plan/bids to the spot market. 
B. Multiple Solution Issue of the Aggregator Optimization with LP Formulation

The multiple solution issue of the aggregator optimization in a LP formulation was highlighted by the discussant in [17] based on the observation of the case study results in [16]. According to that observation, there are an infinite number of optimal solutions in the aggregator optimization problem due to the equal DLMPs at some load points. The multiple solution issue in the aggregator LP optimization is further illustrated bellow.

Assume that there is one EV (or HP) in the distribution network and it is available for energy planning in two periods. It is also assumed that the energy requirement cannot be fulfilled by consuming power in only one period due to the network constraints. For such a case, the DSO optimization is,

$$
\min _{p} c_{1} p_{1}+c_{2} p_{2}
$$

subject to,

$$
\begin{gathered}
D p_{1} \leq f_{1},\left(\lambda_{1}\right) \\
D p_{2} \leq f_{2},\left(\lambda_{2}\right) \\
a_{1} p_{1}+a_{2} p_{2} \geq b,(\mu) \\
p_{1}, p_{2} \geq 0, \quad\left(\varsigma_{1}, \varsigma_{2}\right)
\end{gathered}
$$

Constraints (13) and (14) are network constraints for the two periods, constraint (15) is the energy requirement (derived from (4) and (10), parameter $b$ is the summation of all constants of (4) and (10); the upper limit is ignored for simplicity), and constraint (16) is to set the lower limit of the consumed power $\left(p_{1}, p_{2}\right)$ (the upper limit is ignored for simplicity). Coefficients $a_{1}$ and $a_{2}$ are positive ( $a_{1}=a_{2}=1$ when it is EV).

According to the KKT conditions, the DLMPs are calculated as: (note that $\varsigma_{1}, \varsigma_{2}=0$ and $p_{1}, p_{2}>0$, because the energy requirement cannot be fulfilled by any one of them)

$$
\begin{aligned}
& c_{1}+D^{T} \lambda_{1}=\mu a_{1} \\
& c_{2}+D^{T} \lambda_{2}=\mu a_{2}
\end{aligned}
$$

where the terms $D^{T} \lambda_{1}$ and $D^{T} \lambda_{2}$ are the DTs and should be sent to the aggregator.

The aggregator optimization (no network constraints) is,

$$
\min _{p}\left(c_{1}+\lambda_{1}{ }^{T} D\right) p_{1}+\left(c_{2}+\lambda_{2}{ }^{T} D\right) p_{2}
$$

subject to (15) and (16). It can be seen that such a linear program has an infinite number of optimal solutions due to the proportional coefficients. Hence, the aggregator optimization and the DSO optimization may diverge and the decentralization scheme fails. For instance, the optimal energy plan of the aggregator optimization, where $p_{1}=0$, is infeasible for the DSO optimization because the energy requirement cannot be fulfilled by any one of $p_{1}, p_{2}$, as stated in the assumption.

When there are many flexible demands in the distribution network, the above analysis is still valid, as there is at least one flexible demand behaving like the one in the above example. As such, the decentralized congestion management formulated through LP fails due to degeneracy.

\section{QP Formulation and the Proof of Convergence}

\section{1) DSO Optimization through $Q P$ :}

The DSO optimization in the second step of the procedures in Section IV-A is,

$$
\begin{gathered}
\min _{p_{i, t}, \hat{p}_{i, t}} \sum_{i \in N_{B}, t \in N_{T}} \frac{1}{2} p_{i, t}{ }^{T} B_{i, t} p_{i, t}+\left(c_{t} 1\right)^{T} p_{i, t}+ \\
\frac{1}{2} \hat{p}_{i, t}{ }^{T} B_{i, t} \hat{p}_{i, t}+\left(c_{t} 1\right)^{T} \hat{p}_{i, t}
\end{gathered}
$$

subject to,

$$
\sum_{i \in N_{B}} D E_{i}\left(p_{i, t}+\hat{p}_{i, t}\right) \leq f_{t}, \forall t \in N_{T},\left(\lambda_{t}\right)
$$

together with (4), (5), (10) and (11).

The conventional household demands are assumed to be inflexible. Therefore, they are not included in the objective function (19), but reflected in the line loading limits $f_{t}$, which are the total line capacities excluding the loadings induced by the conventional demands.

The DTs, defined as $D^{T} \lambda_{t}$, will be published by the DSO before the day-ahead market clears. Parameters $c_{t}$ and $\beta_{t}$ used by the DSO are shared with the aggregators since the aggregators need them in their optimization problems.

2) Aggregator Optimization through $Q P$ :

Aggregator $i$ first forms the DLMP for each of his customers, i.e. $c_{t} 1+E_{i}^{T} D^{T} \lambda$.Then, the optimal energy plan of aggregator $i$ can be formulated as,

$$
\begin{aligned}
\min _{p_{i, t}, \hat{p}_{i, t}} \sum_{t \in N_{T}} & \frac{1}{2} p_{i, t}{ }^{T} B_{i, t} p_{i, t}+\left(c_{t} 1+E_{i}^{T} D^{T} \lambda_{t}\right)^{T} p_{i, t}+ \\
& \frac{1}{2} \hat{p}_{i, t}{ }^{T} B_{i, t} \hat{p}_{i, t}+\left(c_{t} 1+E_{i}^{T} D^{T} \lambda_{t}\right)^{T} \hat{p}_{i, t}
\end{aligned}
$$

subject to,

$$
\begin{gathered}
e_{i}^{\min } \leq \sum_{t_{-} \leq t}\left(p_{i, t_{-}}-d_{i, t_{-}}\right)+e_{i 0} \leq e_{i}^{\max }, \forall \mathrm{t} \in \mathrm{N}_{t},\left(\mu_{i, t}^{-}, \mu_{i, t}^{+}\right) \\
p_{i, t}^{\min } \leq p_{i, t} \leq p_{i, t}^{\max } \quad \forall t \in N_{T},\left(\varsigma_{i, t}^{-}, \varsigma_{i, t}^{+}\right) \\
K_{i, t}^{a, \min } \leq \sum_{t_{-} \leq t} A_{i, t, t_{-}} \hat{p}_{i, t_{-}}+u_{i, t} \leq K_{i, t}^{a, \max }, \forall \mathrm{t} \in N_{T},\left(\hat{\mu}_{i, t}^{-}, \hat{\mu}_{i, t}^{+}\right) \\
\hat{p}_{i, t}^{\min } \leq \hat{p}_{i, t} \leq \hat{p}_{i, t}^{\max } \quad t \in N_{T},\left(\hat{\zeta}_{i, t}^{-}, \hat{\zeta}_{i, t}^{+}\right)
\end{gathered}
$$

3) Proof of the Convergence of the DSO Optimization and the Aggregator Optimization through QP:

The KKT conditions of the DSO optimization are,

$$
\begin{gathered}
B_{i, t} p_{i, t}+c_{t} 1+E_{i}^{T} D^{T} \lambda_{t}+\sum_{t_{-} \leq t}\left(\mu_{i, t_{-}}^{+}-\mu_{i, t_{-}}^{-}\right)+\left(\varsigma_{i, t}^{+}-\varsigma_{i, t}^{-}\right) \\
=0, \forall i \in N_{B}, t \in N_{T} \\
B_{i, t} \hat{p}_{i, t}+c_{t} 1+E_{i}^{T} D^{T} \lambda_{t}+\sum_{t_{-} \leq t}\left(\hat{\mu}_{i, t_{-}}^{+}-\hat{\mu}_{i, t_{-}}^{-}\right)+\left(\hat{\zeta}_{i, t}^{+}-\hat{\zeta}_{i, t}^{-}\right) \\
=0, \forall i \in N_{B}, t \in N_{T} \\
\left(\sum_{i} D E_{i} p_{i, t}-f_{t}\right) \cdot \lambda_{t}=0, \forall \mathrm{t} \in N_{T} \\
\left(\sum_{t_{-} \leq t}\left(p_{i, t_{-}}-d_{i, t_{-}}\right)+e_{i, 0}-e_{i}^{\max }\right) \cdot \mu_{i, t}^{+}=0, \forall \mathrm{t} \in \mathrm{N}_{T}, i \in N_{B} \\
\left(\sum_{t_{-} \leq t}\left(p_{i, t_{-}}-d_{i, t_{-}}\right)+e_{i, 0}-e_{i}^{\min }\right) \cdot \mu_{i, t}^{-}=0, \forall \mathrm{t} \in \mathrm{N}_{t}, i \in N_{B} \\
\left(p_{i, t}-p_{i, t}^{\max }\right) \cdot \varsigma_{i, t}^{+}=0, \forall i \in N_{B}, t \in N_{T}
\end{gathered}
$$




$$
\begin{gathered}
\left(p_{i, t}-p_{i, t}^{\min }\right) \cdot \varsigma_{i, t}^{-}=0, \forall i \in N_{B}, t \in N_{T} \\
\left(\sum_{t_{-} \leq t} A_{i, t, t_{-}} \hat{p}_{i, t_{-}}+u_{i, t}-K_{i, t}^{a, \max }\right) \cdot \hat{\mu}_{i, t}^{+}=0, \forall i \in \mathrm{N}_{B}, \mathrm{t} \in N_{T} \\
\left(\sum_{t_{-} \leq t} A_{i, t, t_{-}} \hat{p}_{i, t_{-}}+u_{i, t}-K_{i, t}^{a, \min }\right) \cdot \hat{\mu}_{i, t}^{-}=0, \forall i \in \mathrm{N}_{B}, \mathrm{t} \in N_{T} \\
\left(\hat{p}_{i, t}-\hat{p}_{i, t}^{\max }\right) \cdot \hat{\varsigma}_{i, t}^{+}=0, \forall i \in N_{B}, t \in N_{T} \\
\left(\hat{p}_{i, t}-\hat{p}_{i, t}^{\min }\right) \cdot \hat{\varsigma}_{i, t}^{-}=0, \forall i \in N_{B}, t \in N_{T} \\
\lambda_{t} \geq 0, \forall t \in N_{T} \\
\mu_{i, t}^{+}, \mu_{i, t}^{-}, s_{i, t}^{+}, \varsigma_{i, t}^{-} \hat{\mu}_{i, t}^{+} \hat{\mu}_{i, t}^{-} \hat{\varsigma}_{i, t}^{+}, \hat{\varsigma}_{i, t}^{-} \geq 0, \forall i \in N_{B}, t \in N_{T}
\end{gathered}
$$

together with the constraints (4), (5), (10), (11) and (20).

Similarly, the KKT conditions of the aggregator $i$ optimization are,

$$
\begin{gathered}
B_{i} p_{i, t}+c_{t} 1+E_{i}^{T} D^{T} \lambda_{t}+\sum_{t_{-} \leq t}\left(\mu_{i, t_{-}}^{+}-\mu_{i, t_{-}}^{-}\right)+\left(\varsigma_{i, t}^{+}-\varsigma_{i, t}^{-}\right) \\
=0, \forall t \in N_{T} \\
B_{i} \hat{p}_{i, t}+c_{t} 1+E_{i}^{T} D^{T} \lambda_{t}+\sum_{t_{-} \leq t}\left(\hat{\mu}_{i, t_{-}}^{+}-\hat{\mu}_{i, t_{-}}^{-}\right)+\left(\hat{\varsigma}_{i, t}^{+}-\hat{\varsigma}_{i, t}^{-}\right) \\
=0, \forall t \in N_{T} \\
\left(\sum_{t_{-} \leq t}\left(p_{i, t_{-}}-d_{i, t_{-}}\right)+e_{i, 0}-e_{i}^{\max }\right) \cdot \mu_{i, t}^{+}=0, \forall \mathrm{t} \in \mathrm{N}_{T} \\
\left(\sum_{t_{-} \leq t}\left(p_{i, t_{-}}-d_{i, t_{-}}\right)+e_{i, 0}-e_{i}^{\min }\right) \cdot \mu_{i, t^{-}}^{-}=0, \forall \mathrm{t} \in \mathrm{N}_{t} \\
\left(p_{i, t}-p_{i, t}^{\max }\right) \cdot \varsigma_{i, t}^{+}=0, \forall t \in N_{T} \\
\left(p_{i, t}-p_{i, t}^{\min }\right) \cdot \varsigma_{i, t}^{-}=0, \forall t \in N_{T} \\
\left(\sum_{t_{-} \leq t} A_{i, t, t_{-}} \hat{p}_{i, t_{-}}+u_{i, t}-K_{i, t}^{a, \max }\right) \cdot \hat{\mu}_{i, t}^{+}=0, \forall \mathrm{t} \in N_{T} \\
\left(\sum_{t_{-} \leq t} A_{i, t, t_{-}} \hat{p}_{i, t_{-}}+u_{i, t}-K_{i, t}^{a, \min }\right) \cdot \hat{\mu}_{i, t}^{-}=0, \forall \mathrm{t} \in N_{T} \\
\left(\hat{p}_{i, t}-\hat{p}_{i, t}^{\max }\right) \cdot \hat{\varsigma}_{i, t}^{+}=0, \forall t \in N_{T} \\
\left(\hat{p}_{i, t}-\hat{p}_{i, t}^{\min }\right) \cdot \hat{\zeta}_{i, t}^{-}=0, \forall t \in N_{T}
\end{gathered}
$$

together with (22)-(25) and (38).

It can be seen that the objective function (19) of the DSO problem is a quadratic function with all quadratic terms being positive and no cross terms. Therefore, the Hessian matrix can be found by observation. Particularly, it is a diagonal matrix with the elements being the coefficients of the quadratic terms in (19), which are all positive. A diagonal matrix with all elements being positive is a positive definite matrix; therefore, the Hessian matrix of (19) is positive definite.

Since the objective function (19) is a quadratic function with positive definite Hessian matrix and all the constraints, i.e. (4), (5), (10), (11) and (20) are affine functions, the DSO optimization problem is a strictly convex QP problem, which has a unique minimizer [21] assuming the problem is feasible. Moreover, the KKT conditions of the DSO optimization problem are necessary and sufficient [21].

Similarly, it can be inferred from (21)-(25) that each aggregator optimization problem is also a strictly convex QP problem. Therefore, each of them has a unique minimizer and the KKT conditions are necessary and sufficient.

Now, suppose

$$
\left(p_{i, t}{ }^{*}, \hat{p}_{i, t}{ }^{*}, \lambda_{t}{ }^{*}, \mu_{i, t}^{+*}, \mu_{i, t}^{-*},{ }_{i, t}^{+*}, S_{i, t}^{-*}, \hat{\mu}_{i, t}^{+*}, \hat{\mu}_{i, t}^{-*}, \hat{\varsigma}_{i, t}^{+*}, \hat{\varsigma}_{i, t}^{-*}\right)
$$

is a solution of the KKT conditions of the DSO problem ((4), (5), (10), (11), (20) and (26)-(38)), implying that $\left(p_{i, t}{ }^{*}, \hat{p}_{i, t}{ }^{*}\right)$ is a solution of the problem. By comparing the KKT conditions, it can be seen that, with respect to aggregator $i$,

$$
\left(p_{i, t}{ }^{*}, \hat{p}_{i, t}{ }^{*}, \mu_{i, t}^{+*}, \mu_{i, t}^{-*}, \varsigma_{i, t}^{+*}, \varsigma_{i, t}^{-*}, \hat{\mu}_{i, t}^{+*}, \hat{\mu}_{i, t}^{-*}, \hat{\varsigma}_{i, t}^{+*}, \hat{\varsigma}_{i, t}^{-*}\right)
$$

is also satisfying (22)-(25) and (38)-(48), i.e. the KKT conditions of the aggregator problem. This means $\left(p_{i, t}{ }^{*}, \hat{p}_{i, t}{ }^{*}\right)$ is also a solution of the aggregator problem. Because any solution of the DSO problem must satisfy the KKT conditions of it, it can be concluded that any solution of the DSO problem is also a solution to the aggregator problem.

On the other hand, a solution that satisfies the KKT conditions of the aggregator problems does not necessarily satisfy the KKT conditions of the DSO problem, because the switching condition (28) of the DSO problem is not respected by the aggregator problems. However, due to the uniqueness of the solution to the DSO problem and the aggregator problems, any solution of the aggregator problems must also be a solution of the DSO problem. This can be proven by contradiction.

Suppose $\left(p_{i, t}{ }^{* *}, \hat{p}_{i, t}{ }^{* *}\right)$ is a solution of the aggregator problems but not to the DSO problem. Suppose $\left(p_{i, t}{ }^{*}, \hat{p}_{i, t}{ }^{*}\right)$ is a solution to the DSO problem. Then, according to the previous conclusion, $\left(p_{i, t}{ }^{*}, \hat{p}_{i, t}{ }^{*}\right)$ is also a solution to the aggregator problems. Due to the uniqueness of the aggregator problems, there is $\left(p_{i, t}{ }^{*}, \hat{p}_{i, t}{ }^{*}\right)=\left(p_{i, t}{ }^{* *}, \hat{p}_{i, t}{ }^{* *}\right)$ and it contradicts the assumption that $\left(p_{i, t}^{* *}, \hat{p}_{i, t}{ }^{* * *}\right)$ is not a solution to the DSO problem. Therefore, it can be concluded that any solution to the aggregator problems is also a solution to the DSO problem. Based on the above conclusions, the DSO problem and the aggregator problems are equivalent.

\section{CASe Studies}

Case studies were conducted using the Danish driving pattern and the Bus 4 distribution system of the Roy Billinton Test System (RBTS) [22]. The details of the case studies are presented in this section.

\section{A. Grid Data}

The single line diagram of the Bus 4 distribution network is shown in Fig. 3. Line segments of the feeder one are labeled in Fig. 3, among which L2, L4, L6, L8, L9, L11, and L12 refer to the transformers connecting the corresponding load points (LP1 to LP7). The study is focused on this feeder because of its diversity: 5 residential load points with different peak conventional demands and two commercial load points. The detailed data of these load points are listed in Table I. The peak conventional demands of residential customers are assumed to occur at 18:00 when people come home and start cooking (shown in Fig. 5). 


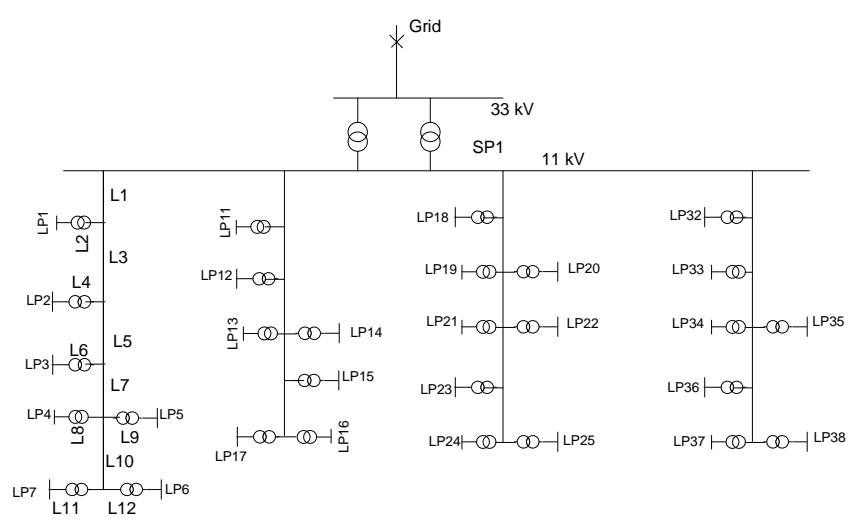

Fig. 3. Single line diagram of the distribution network

TABLE I

LOAD POINT DATA

\begin{tabular}{llll}
\hline $\begin{array}{c}\text { load } \\
\text { points }\end{array}$ & $\begin{array}{c}\text { customer } \\
\text { type }\end{array}$ & $\begin{array}{c}\text { peak conv. } \\
\text { load per } \\
\text { point (kW) }\end{array}$ & $\begin{array}{c}\text { number of } \\
\text { customers } \\
\text { per point }\end{array}$ \\
\hline LP1-LP4 & residential & 886.9 & 200 \\
\hline LP5 & residential & 813.7 & 200 \\
\hline LP6,LP7 & commercial & 671.4 & 10 \\
\hline
\end{tabular}

\section{B. EV and HP Data}

The key parameters of EVs and HPs are listed in Table II. The EV availability shown in Fig. 4 is from the driving pattern study in [23]. The household area is a random number between 100 and $200\left(\mathrm{~m}^{2}\right)$.

TABLE II

KEY PARAMETERS OF EVS AND HPs ([23], [25])

\begin{tabular}{ll}
\multicolumn{1}{c}{ parameter } & \multicolumn{1}{c}{ value } \\
\hline EV battery size & $25 \mathrm{kWh}$ \\
\hline Peak charging power & $11 \mathrm{~kW}(3$ phase $)$ \\
\hline Energy consumption per km & $150 \mathrm{Wh} / \mathrm{km}$ \\
\hline Minimum SOC & $20 \%$ \\
\hline Maximum SOC & $85 \%$ \\
\hline Average driving distance & $40 \mathrm{~km}$ \\
\hline COP of HP & 2.3 \\
\hline Min Temp. of the House & $20{ }^{\circ} \mathrm{C}$ \\
\hline Max Temp. of the House & $24{ }^{\circ} \mathrm{C}$ \\
\hline
\end{tabular}

\section{Case Study Results}

In the case study, it is assumed that there are two aggregators. The aggregator 'aag1' has contracts with 40 customers per load point while the other has contracts with the rest 160 customers per load point. The line loading limits of all line segments are listed in Table III, which are higher than the peak conventional demands but lower than the peak demands including EVs and HPs.

The simulation was carried out using the General Algebraic Modeling System (GAMS) optimization software [24] alt- hough many other tools can be used such as QUADPROG in MATLAB, Gurobi and AMPL. Firstly, the DSO optimization problem was carried out and the results are shown in Fig. 5 (due to the space limitation, only the results of line L2-L4 were plotted). Because the line loading limits are respected in the optimization, the line loadings of all line segments are lower than the limits.

It can be seen from Fig. 5 that the line loadings reach (but not exceed) the limits at hour 16-18 (only line L2) and hour 23-24. This means that the corresponding inequality constraints of the optimization problem are 'active' and the Lagrange multipliers of these constraints are positive. Therefore, according to the DLMP calculation method described in Section IV-C, the DLMPs are higher than the base price (shown in Fig. 6 and Table IV). The prices of LP1 at hour 17-18 are very high and are chopped in Fig. 6 (they can be found in Table IV) in order to have a better illustration of DLMPs of other hours. The high prices of LP1 at hour 17-18 can be explained by analyzing the nature of the congestion caused by HPs. HPs are less sensitive to the prices compared to EVs because of the significant thermal leakages of the households; therefore, higher DLMPs are required to solve the congestion caused by them.

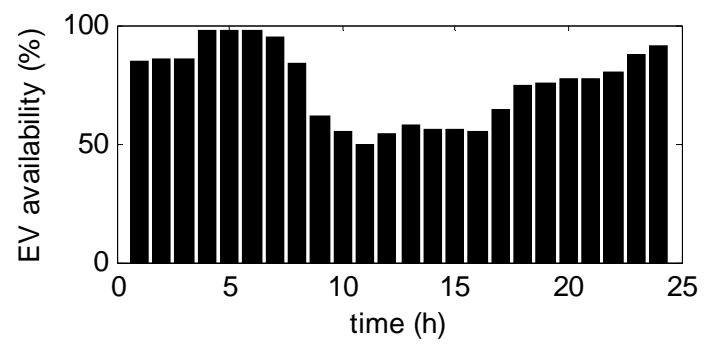

Fig. 4. EV availability

TABLE III

LINE LOADING LIMIT

\begin{tabular}{clllll}
\hline line & L2 & L3 & L4 & L8 & L9 \\
\hline $\begin{array}{l}\text { limit } \\
(\mathbf{k W})\end{array}$ & 1400 & 7000 & 1700 & 1600 & 1500 \\
\hline
\end{tabular}

TABLE IV

DLMPs (DKK/KWH) DUE TO MULTIPLE CONGESTIONS ON L2, L3, L4, L8 AND L9 ('-' MEANs Equal to BASE PRICE)

\begin{tabular}{cllllll}
\hline time & 5 & 16 & 17 & 18 & 23 & 24 \\
\hline $\begin{array}{c}\text { base } \\
\text { price }\end{array}$ & 0.3012 & 0.3884 & 0.3513 & 0.3313 & 0.2941 & 0.2241 \\
\hline LP1 & - & 0.5611 & 1.1006 & 2.4335 & 0.3012 & 0.3012 \\
\hline LP2 & - & - & - & - & - & 0.2940 \\
\hline LP3 & - & - & - & - & - & 0.2937 \\
\hline LP4 & - & - & - & - & 0.3006 & 0.3006 \\
\hline LP5 & - & - & - & - & 0.3008 & 0.3008 \\
\hline
\end{tabular}




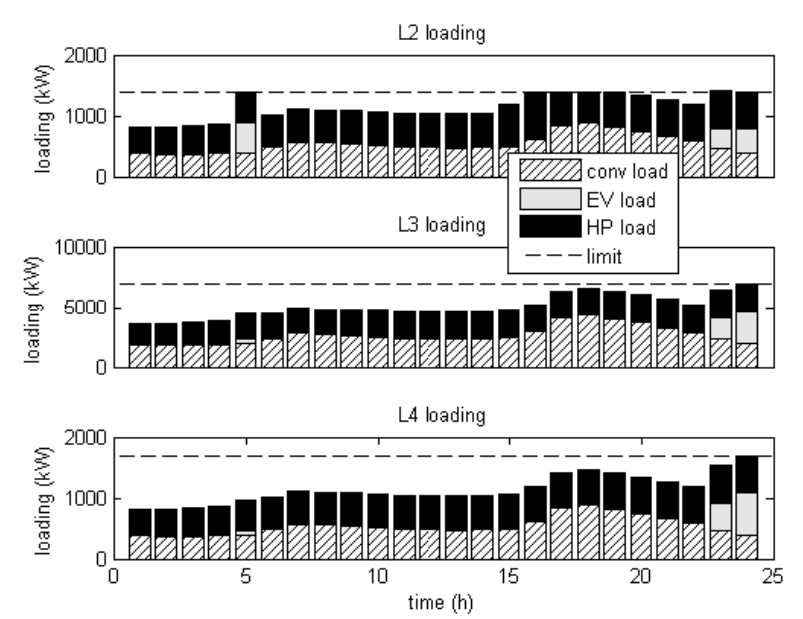

Fig. 5. Line loading of the DSO problem

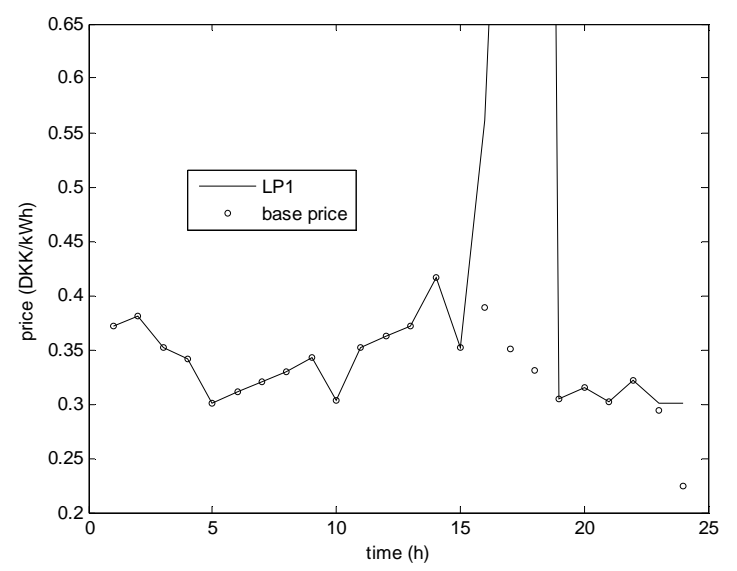

Fig. 6. System prices and DLMPs at LP1

Secondly, the aggregator optimization was performed. Two aggregators carried out their own optimization problem independently.

In order to clearly show the effect of the DLMP, two case studies were conducted. In Case One, the DLMP was not applied; in Case Two, the DLMP was applied.

As expected, when the DLMP is not applied, congestions occur at 24:00 and 18:00 (shown in Fig. 7). At 24:00, because the system price is the lowest, every EV wants to charge its battery as long as it is available for charging. The simultaneous charging leads to the very high peak. Overloading of line L2 at 18:00, however, is not due to the low price. In fact, it is the peak conventional demand that has consumed most of the capacity of the line and the available capacity is not enough for the HP demands.

When the DLMP is applied, the congestions are alleviated (shown in Fig. 8). Due to the posed DTs, the DLMP at load points LP1 at 24:00 is as attractive as the ones at 23:00 and 5:00. Therefore, the EV charging demands are spread at those hours and the resulted peak is not higher than the limits. The previous congestion of line L2 at 18:00 also disappears due to the DLMP. The DLMP at LP1 at 18:00 is so high that the HPs choose to produce more heat before $18: 00$ and due to the dy- namics of the thermal objects (house inside air, house structure), the temperature at 18:00 is maintained between the lower and upper limits. Hence, the HP demands are shifted to the previous hours when the conventional demands are lower enough to accommodate them.
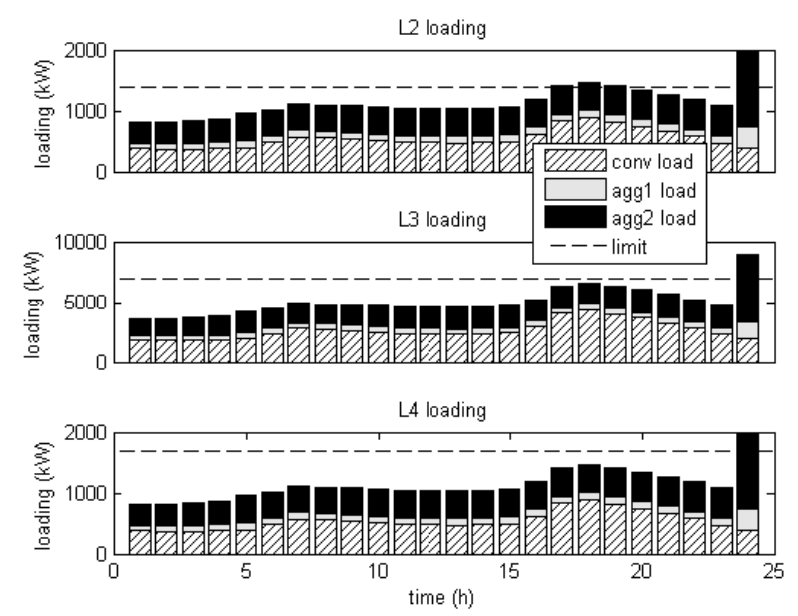

Fig. 7. Line loading without DLMP
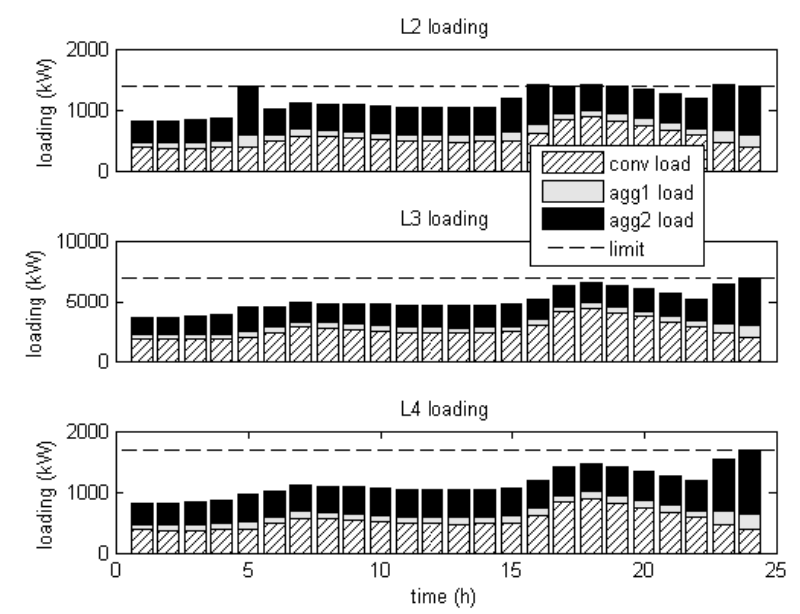

Fig. 8. Line loading with DLMP

In order to illustrate the divergence issue that might occur with the LP formulation, a simulation was conducted where the price sensitive part was excluded. Without the price sensitive part, the DSO optimization problem and the aggregator optimization problems are LPs. The DLMPs were calculated and shown in Table V. It can be seen that the DLMPs of LP1 are the same at time 5, 23 and 24 hour. This will lead to infinite solutions to the aggregator problems. As a result, the aggregator may not act as the DSO expects. This is confirmed by the simulation results in Fig. 9 and Fig. 10. In Fig. 9, for the DSO optimization, there is no congestion, however, in Fig. 10, for the aggregator optimization, congestions occur at line L2; loading of line L3 at 5 hour is different. 


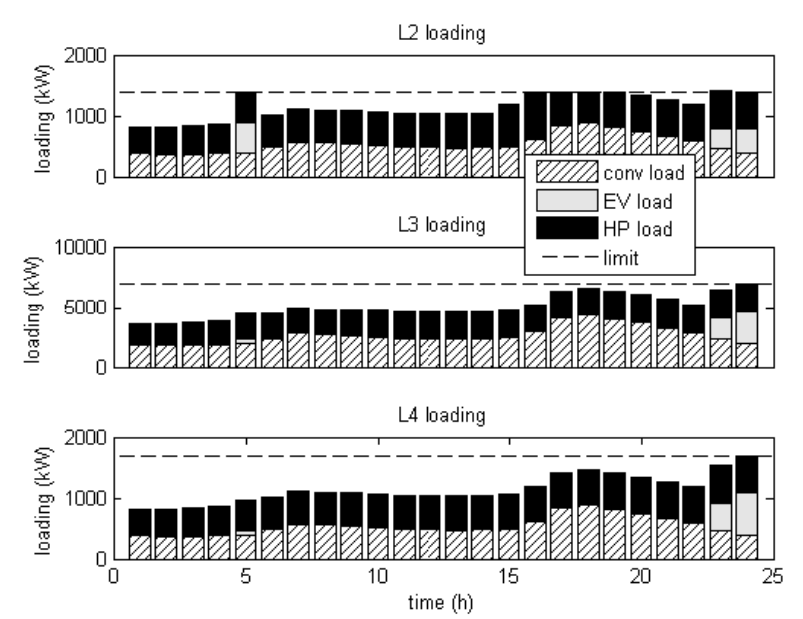

Fig. 9. Line loading of the DSO problem excluding quadratic terms
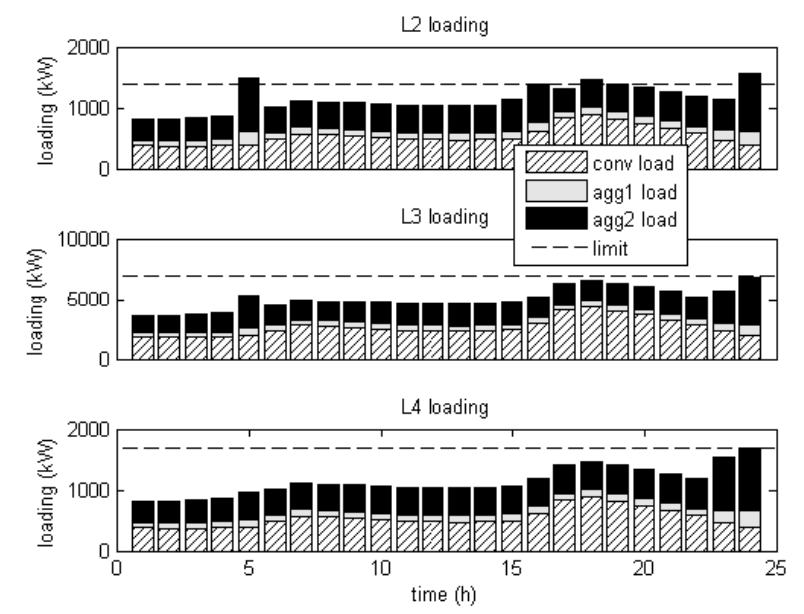

Fig. 10. Line loading of the aggregator problems excluding quadratic terms

TABLE V

DLMPS (DKK/KWH) WITH MULTIPLE CONGESTIONS AT L2, L3, L4, L8 AND L9 ('-': EQ. TO BASE PRICE), CALC. WITHOUT QuAdRATIC TERMS

\begin{tabular}{cllllll}
\hline time & 5 & 16 & 17 & 18 & 23 & 24 \\
\hline $\begin{array}{c}\text { base } \\
\text { price }\end{array}$ & 0.3012 & 0.3884 & 0.3513 & 0.3313 & 0.2941 & 0.2241 \\
\hline LP1 & - & 0.5605 & 1.0984 & 2.4267 & 0.3012 & 0.3012 \\
\hline LP2 & - & - & - & - & - & 0.2941 \\
\hline LP3 & - & - & - & - & - & 0.2941 \\
\hline LP4 & - & - & - & - & 0.3012 & 0.3012 \\
\hline LP5 & - & - & - & - & 0.3012 & 0.3012 \\
\hline
\end{tabular}

\section{CONCLUSIONS AND FUTURE WORK}

Though the DLMP and DT concepts are efficient in alleviating congestions in distribution networks with high penetration of flexible demands, the formulation of the decentralized aggregator optimization must be carefully handled. With a LP formulation of the aggregator optimization, there might be multiple solutions to the decentralized aggregator optimization due to degeneracy. The multiple solutions to the aggregator optimization may cause the centralized DSO optimization and the decentralized aggregator optimization to diverge, and the decentralized congestion management approach to fail.
The multiple solution issue of the aggregator optimization is addressed in this paper by introducing price sensitivity which leads to strictly convex QP formulation for both the DSO optimization and the aggregator optimization. The equivalence of the centralized DSO optimization and the decentralized aggregator optimization with the QP formulation is proven which ensures that the aggregators act as the DSO expects. The case study results have demonstrated the equivalence of the DSO optimization and the aggregator optimization with a strictly convex QP formulation, and the efficacy of the DLMP through QP for congestion management.

For future work, more practical features of the distribution network can be considered, such as high $\mathrm{R} / \mathrm{X}$ ratio, losses, single phase loads and unbalance. It is interesting to study how these factors will affect the DLMP for congestion management. In addition to the line loading constraints, voltage constraints shall also be studied in the future work.

\section{REFERENCES}

[1] The Danish Government (2011, Feb.). Energy strategy 2050. Copenhagen, Denmark. [Online]. Available: http://www.ens.dk/sites/ens.dk/files/dokumenter/publikationer/download s/energy_strategy_2050.pdf (accessed on 04-Aug-2014).

[2] G. Delille, B. Francois, and G. Malarange, "Dynamic frequency control support by energy storage to reduce the impact of wind and solar generation on isolated power system's inertia," IEEE Trans. Sustain. Energy, vol. 3, no. 4, pp. 931-939, Oct. 2012.

[3] Z. H. Rather, Z. Chen, and P. Thogersen, "Impact of wind energy integration on reactive power reserve and its smart solution: A danish power system case study,” in Proc. 2012 IEEE International Conference on Power System Technology (POWERCON), pp. 1-6.

[4] J. R. Pillai and B. Bak-Jensen, "Impacts of electric vehicle loads on power distribution systems," in Proc. 2010 IEEE Vehicle Power and Propulsion Conference, pp. 1-6.

[5] B. Biegel, P. Andersen, J. Stoustrup, and J. Bendtsen, “Congestion management in a smart grid via shadow prices,” in Proc. 2012 8th IFAC Symposium on Power Plant and Power System Control, pp. 518-523.

[6] J. Hu, S. You, M. Lind, and J. Ostergaard, "Coordinated charging of electric vehicles for congestion prevention in the distribution grid," IEEE Trans. Smart Grid, vol. 5, no. 2, pp. 703-711, Mar. 2014.

[7] N. O'Connell, Q. Wu, J. Østergaard, A. H. Nielsen, S. T. Cha, and Y. Ding, "Day-ahead tariffs for the alleviation of distribution grid congestion from electric vehicles,” Electr. Power Syst. Res., vol. 92, pp. 106-114, 2012.

[8] A. R. Malekpour, A. R. Seifi, M. R. Hesamzadeh, and N. Hosseinzadeh, "An optimal load shedding approach for distribution networks with DGs considering capacity deficiency modelling of bulked power supply,” in Proc. 2008 IEEE Power Engineering Conference, pp. 1-7.

[9] M. Alonso, H. Amarís, C. Álvarez, and R. Albarracín, "Reactive power planning in distribution networks with distributed generation," in Proc. 2010 7th Mediterranean Conference and Exhibition on Power Generation, Transmission, Distribution and Energy Conversion, pp. 152-152.

[10] R. Bohn, M. Caramanis, and F. Schweppe, "Optimal pricing in electrical networks over space and time,” RAND J. Econ., vol. 15, no. 3, pp. 360376, 1984.

[11] P. M. Sotkiewicz and J. M. Vignolo, "Nodal pricing for distribution networks: efficient pricing for efficiency enhancing DG,” IEEE Trans. Power Syst., vol. 21, no. 2, pp. 1013-1014, May 2006.

[12] R. K. Singh and S. K. Goswami, "Optimum allocation of distributed generations based on nodal pricing for profit, loss reduction, and voltage improvement including voltage rise issue,” Int. J. Electr. Power Energy Syst., vol. 32, no. 6, pp. 637-644, Jul. 2010.

[13] F. Meng and B. H. Chowdhury, "Distribution LMP-based economic operation for future smart grid," in Proc. 2011 IEEE Power and Energy Conference at Illinois, pp. 1-5.

[14] G. T. Heydt, B. H. Chowdhury, M. L. Crow, D. Haughton, B. D. Kiefer, F. Meng, and B. R. Sathyanarayana, "Pricing and control in the next 
generation power distribution system,” IEEE Trans. Smart Grid, vol. 3, no. 2, pp. 907-914, Jun. 2012

[15] K. Shaloudegi, N. Madinehi, S. H. Hosseinian, and H. A. Abyaneh, “A novel policy for locational marginal price calculation in distribution systems based on loss reduction allocation using game theory," IEEE Trans. Power Syst., vol. 27, no. 2, pp. 811-820, May 2012.

[16] R. Li, Q. Wu, and S. S. Oren, "Distribution locational marginal pricing for optimal electric vehicle charging management," IEEE Trans. Power Syst., vol. 29, no. 1, pp. 203-211, Jan. 2014.

[17] W. Liu and F. Wen, "Discussion on 'Distribution Locational Marginal Pricing for Optimal Electric Vehicle Charging Management',’ IEEE Trans. Power Syst., vol. 29, no. 4, pp. 1866-1866, Jul. 2014.

[18] R. A. Verzijlbergh, Z. Lukszo, and M. D. Ilic, "Comparing different EV charging strategies in liberalized power systems,” in Proc. 2012 9th International Conference on the European Energy Market, pp. 1-8.

[19] R. A. Verzijlbergh, L. J. De Vries, and Z. Lukszo, "Renewable energy sources and responsive demand. Do we need congestion management in the distribution grid?,” IEEE Trans. Power Syst., vol. PP, no. 99, pp. 110, Feb. 2014.

[20] P. Bacher and H. Madsen, "Identifying suitable models for the heat dynamics of buildings,” Energy Build., vol. 43, no. 7, pp. 1511-1522, Jul. 2011.

[21] S. Boyd and L. Vandenberghe, Convex Optimization. New York: Cambridge University Press, 2004, p. 727.

[22] R. N. Allan, R. Billinton, I. Sjarief, L. Goel, and K. S. So, “A reliability test system for educational purposes-basic distribution system data and results,” IEEE Trans. Power Syst., vol. 6, no. 2, pp. 813-820, May 1991.

[23] Q. Wu, A. H. Nielsen, J. Østergaard, F. Marra, and C. Træholt, "Driving pattern analysis for electric vehicle (EV) grid integration study," in Proc. 2010 IEEE PES Innovative Smart Grid Technologies Conference Europe (ISGT Europe), pp. 1-6.

[24] R. E. Rosenthal (2014, Jul.). GAMS - A user's guide. GAMS Development Corporation, Washington DC, USA. [Online]. Available: http://www.gams.com/dd/docs/bigdocs/GAMSUsersGuide.pdf (accessed on 04-Aug-2014).

[25] J. M. Nyers and A. J. Nyers, "COP of heating-cooling system with heat pump," in Proc. 2011 IEEE 3rd International Symposium on Exploitation of Renewable Energy Sources (EXPRES), pp. 17-21. 